\title{
Leishmanicidal activity of Artemisinin against cutaneous Leishmaniasis, in Vitro
}

\author{
Ghuffran Muhammed Hassan Hayder Z. Ali \\ Department of Biology / College of Science / University of Baghdad
}

E-mail : guffran_bio@yahoo.com, h.z.ali2010@gmail.com

\begin{abstract}
Background: Cutaneous leishmaniasis (CL) is a neglected disease in tropical countries, including Iraq. Several studies have sought to examine chemotherapies for leishmaniasis treatment but most of them are of toxic and/or undesirable side effect, therefore, the need for investigating new fewer toxic therapies is essential.

Aim of study: In this study, the cytotoxic effect of Artemisinin (ART), a novel herbal compound, was screened against the two forms, promastigotes and amastigotes, of the Iraqi isolate of Leishmania tropica, the causative agent of Baghdad boil.

Material and methods: Different concentrations $(1000,500,250,125,62.5,31.25,15.6$ and 7.8) $\mu \mathrm{M}$ of Artemisinin were screened to investigate the leishmanicidal activity of the herbal compound against the two forms of the parasite along three times of follow up $(24,48,72)$ hour using MTT cytotoxicity assay.

Results: The results showed that growth rate and cell viability were significantly decreased at all studied concentrations. The $\mathrm{IC}_{50}$ was measured after 72 hours of follow up and was $2.625 \mu \mathrm{M}$ and $2.636 \mu \mathrm{M}$ for promastigotes and amastigotes, respectively.

Conclusion: These findings approved the leishmanicidal efficacy of Artemisinin against the of $L$. tropica and can be further studied to screen its effectiveness in vivo for exploring a safer herbal drug for treatment of cutaneous leishmaniasis.
\end{abstract}

Key words: Artemisinin ART, Leishmniasis , in vitro, MTT.

\section{Introduction}

Leishmaniasis is one of the most serious epizootic diseases according to the World Health Organization (WHO) (1). Leishmaniasis is a crucial public health problem caused by Leishmania spp., class of Kinetoplastida, it affects 98 countries worldwide an estimated 1.3 million new cases and 20000 to 30000 deaths are reported annually around 310 million people are at risk of an infection (2). Cutaneous leishmaniasis, the skin form of the disease, is an endemic disease in Middle Eastern, such as Syria, Iraq, Kingdom Saudi Arabia, and Jordan and it is still considered as an essential health problem which requires international awareness (3). The drug favorite for therapy of CL. is Glucantime and Pentostam. Both of them possess toxic side effects. Therefore, scientists are evolving less toxic new therapies with minimal toxicity $(4,5)$. Miltefosine and paromomycin are two medications that have been entered more newly for the therapy of leishmaniasis disease (6). However, long-term medication with miltefosine's long half-life (about $152 \mathrm{~h}$ ) can enhance early starter of drug resistance, and potential teratogenic and abortifacient effects limit its prescription through gestation $(7,8)$. In vitro tests have led to the emergence of paromomycin-resistant, miltefosine resistant (9) meglumine antimoniate-resistant (10) and pentamidine-resistant (11). Artemisinin and its derivatives represent a very important new class of antimalarials (12). Artemisinin and aqueous extract of Artemisia sieberi are of plant origin. Artemisinin is derived from a medicinal herb called qinghazo (sweet wormwood) or Artemisia annиa and is still obtained from this plant. Artemisinin is relatively facilely purified after extraction from plants (13). Following their discovery and development of antimalarial drugs by Tu Youyou's group in the 1970s (14). Artemisinin and its derivatives have been investigated in treating parasitic diseases or parasitic infections caused by protozoan parasites including Leishmania spp., Trypanosoma spp., Toxoplasma gondii, Neospora caninum, Eimeria tenella, Acanthamoeba castellanii, Naegleria fowleri, Cryptosporidium parvum, Giardia lamblia, and Babesia spp. (15). They are efficient in inhibiting the parasite metabolism while showing limited adverse effects on the host, indicating a higher safety index of the drugs. A large number of in-vitro or ex-vivo studies have shown that ART and its derivatives have activities in controlling the parasites, and the herbal compound shown effective against the protozoan (16). 


\section{Material and Methods}

Artemisinin $\left(\mathrm{C}_{15} \mathrm{H}_{22} \mathrm{O}_{5}\right)$ was purchased from TOCRIC biotechne (England) and dissolved according to the manufacturer's protocol. In brief, $3 \mathrm{mg}$ of artemisinin was dissolved in $500 \mu \mathrm{L}$ of Dimethyl sulfoxide (DMSO). From the stock solution, different concentrations of ART were prepared as following (500, 400, $300,200,100,50$ and 10) $\mu \mathrm{M}$.

\section{Leishmania tropica isolate culture}

L. tropica was isolated from a patient in AL-Karama hospital (Baghdad city), a patient diagnosed with Cutaneous Leishmaniasis and a sample was taken from a hand lesion (17). Procyclic promastigotes of $L$. tropica were cultured in M199 medium (Sigma Aldrich St. Louis, MO, USA). The medium was prepared according to manufacturer's procedure at $\mathrm{pH} 7.4$ supplemented with $10 \%$ heat-inactivated fetal bovine serum, $100 \mathrm{IU} / \mathrm{ml}$ of penicillin and $100 \mu \mathrm{g} / \mathrm{ml}$ of streptomycin, culture was incubated at $26^{\circ} \mathrm{C}$ For three days to enable proliferation of promastigotes into $\log$ phase (18). The production of axenic amastigotes was induced by transferring the procyclic promastigotes of $5 \times 106$ parasite/ml to a new RPMI140 medium

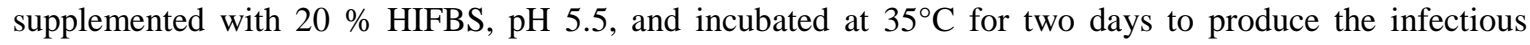
metacyclic promastigotes (19).

\section{Cytotoxicity assay (Colorimetric MTT assay)}

Promastigotes and amastigotes were cultured in plates of 96 wells flat bottom, with the different concentrations: $(1000,500,250,125,62.5,31.25,15.6$ and 7.8) $\mu \mathrm{M}$, each concentration was made in triplicates and each plate was incubated at $26^{\circ} \mathrm{C}$ and $35^{\circ} \mathrm{C}$ for promastigotes and amastigotes, respectively. After 24, 48 and 72 hours of incubation, $20 \mu \mathrm{L}$ of MTT solution was added to each well and the plates was incubated at $26^{\circ} \mathrm{C}$ and $35^{\circ} \mathrm{C}$. The medium was aspirated and the formed formazan crystals were solubilized by adding $50 \mu \mathrm{L}$ DMSO per well for $10 \mathrm{~min}$. Finally, the intensity of the dissolved formazan crystals was quantified by ELISA reader at $620 \mathrm{~nm}(20)$.

\section{Statistical Analysis}

To determine the significant differences between means of control and test values for each concentration after time $(24,48$, and 72$) \mathrm{h}$, using t-test and Different between means have analyzed at $(\mathrm{p} \leq 0.05)$ and expressed as (Mean \pm SE) (21).

\section{Results and Discussion}

The results of cytotoxicity revealed the activity of Artemisinin to inhibit parasite's growth and limit the proliferation, difference of colorimetric absorption was detected. Statistically, there was a significant $(\mathrm{p} \leq$ 0.05 ) difference in absorption of microtiter- plate reader data, between test and control for promastigotes at all concentrations (1000, 500, 250, 125.5, 62.5, 31.25, 15.6 and 7.8) $\mu \mathrm{M}$ after 24,48 and 72 hours of followup. Moreover, the effect was most apparent after 72 hours of treatment. According to the cytotoxicity results above, the $\mathrm{IC}_{50}$ was calculated along the three times of follow up and demonstrated a time-dependent inhibition of the parasite growth in which the $\mathrm{IC}_{50}$ value was only measurable after 72 hours and it was $421.743 \mu \mathrm{M}$.

Cell viability was calculated by plotting the percentages of parasite viability against logarithm Artemisinin concentrations. Results were compared between test and control for all Artemisinin log concentrations as means \pm standard deviation (SE) (21). The percentage of promastigotes viability for the treated groups for all concentration was more than $50 \%$ after 24 - and 48 -hours treatment, therefore no $\mathrm{IC}_{50}$ could be calculated; while after 72 hours treatment, the cell viability of test group dropped below $50 \%$. The mean of cell viability measured at the highest concentration of $1000 \mu \mathrm{M}$ was $(76.61,70.03,41.71) \%$ after 24,48 and 72 hours, respectively figure 1,2 and 3 . Furthermore, the mean of cell viability measured at the lowest concentration of $7.8 \mu \mathrm{M}$ was $(94.76,92.67,92.05) \%$ after 24,48 and 72 hours. 


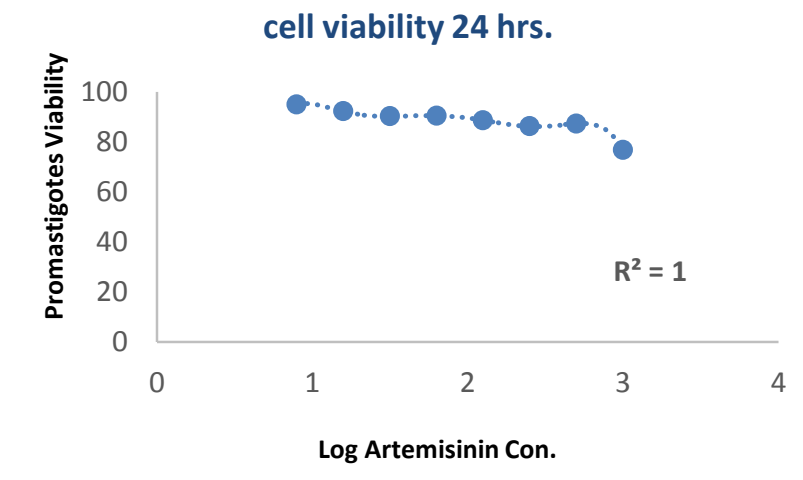

Figure (1): Cell viability of $L$. tropica

promastigotes treated with Artemisinin, after 24 hours of incubation

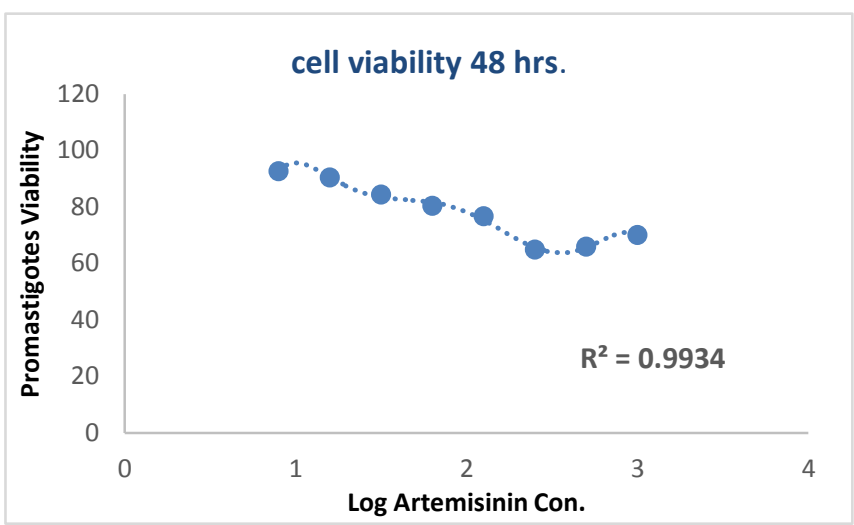

Figure (2): Cell viability of $L$. tropica

promastigotes treated with Artemisinin, after 48 hours of incubation

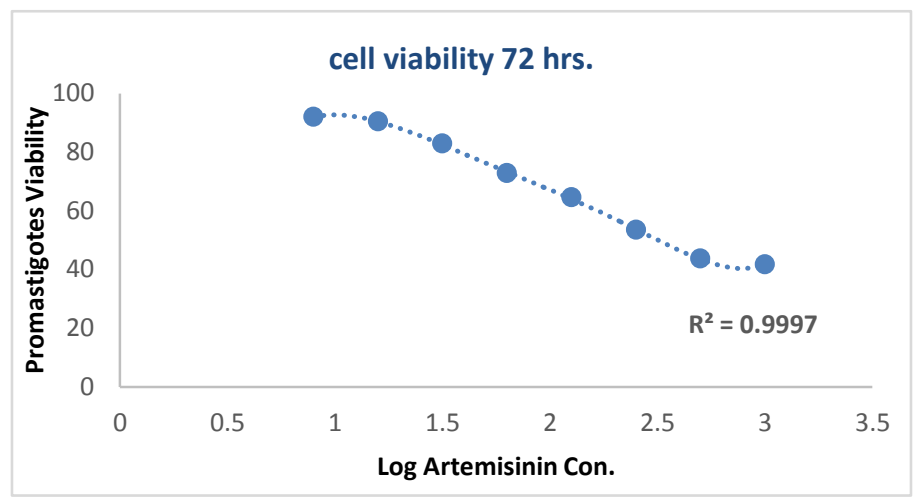

Figure (3): Cell viability of $L$. tropica

promastigotes treated with Artemisinin, after

72 hours of incubation

For amastigotes screening, results have shown that the Artemisinin had inhibited amastigotes proliferation. Statistically, there was a significant $(\mathrm{p} \leq 0.05)$ differences of absorption of microtiter- plate reader data between treated groups and control for amastigotes at all studied concentrations $(1000,500,250,125.5$, $62.5,31.25,15.6$ and 7.8$) \mu \mathrm{M}$ after 24,48 and 72 hours of follow-up.

The percentage of cell viability of amastigotes for test group was more than $50 \%$ after 24 and 48hours incubation, therefore no $\mathrm{IC}_{50}$ could be calculated. While after 72 hours treatment, cell viability of test group dropped below $50 \%$. $\mathrm{IC}_{50}$ was detectable after 72 hours and calculated as $432.661 \mu \mathrm{M}$. The mean of cell viability measured at the highest concentration of $1000 \mu \mathrm{M}$ was $(68.83,62.09,31.26) \%$ after 24,48 and 72 hours, respectively. Furthermore, the mean of cell viability measured at the lowest concentration of $7.8 \mu \mathrm{M}$ was $(88.24,84.47,82.62) \%$ after 24, 48 and 72 hours, respectively. Figure (5,6 and 7), Figure(4,5) and(6). 


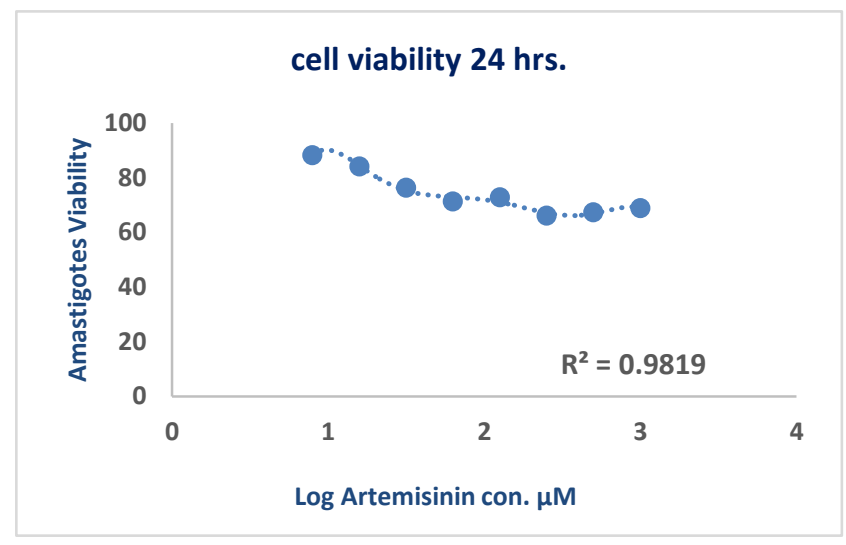

Figure (4): Cell viability of $L$. tropica amastigotes treated with Artemisinin, after 24 hours of incubation

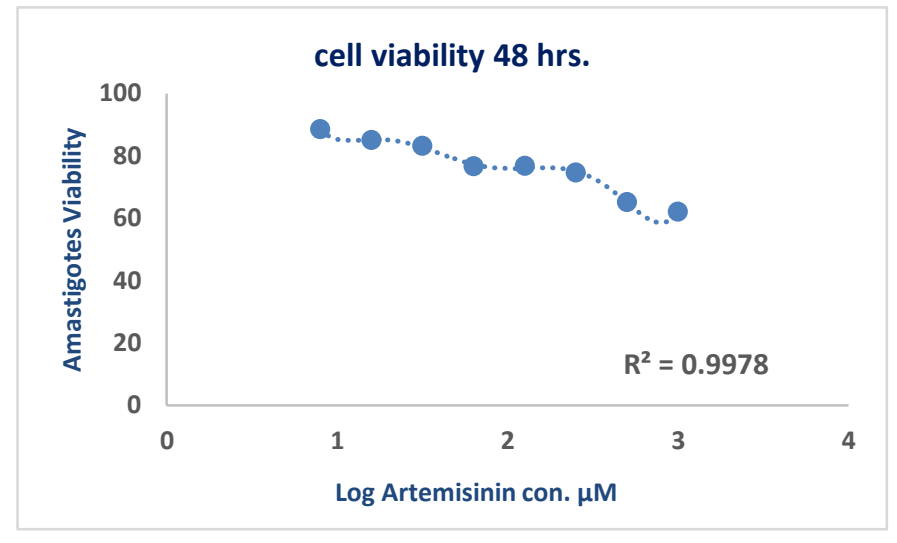

Figure (5): Cell viability of $L$. tropica amastigotes treated with Artemisinin, after48 hours of incubation

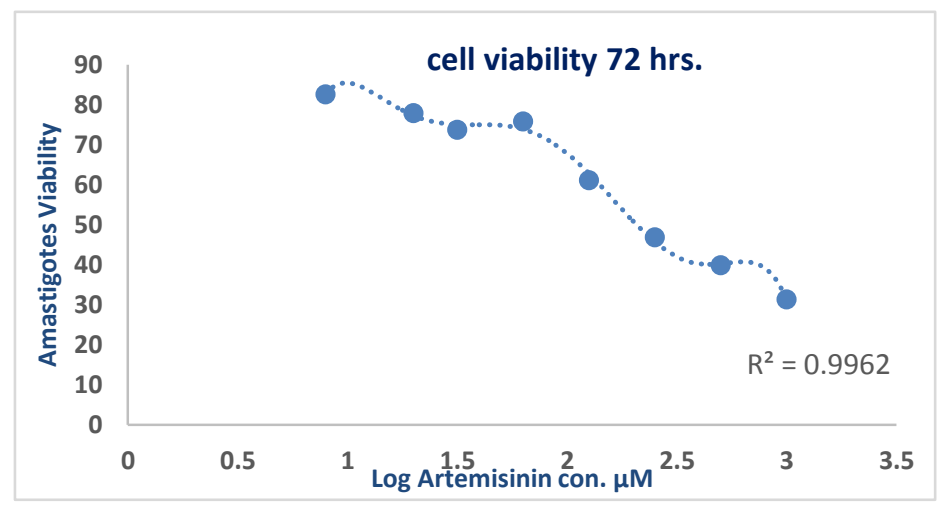

Figure (6): Cell viability of $L$. tropica

amastigotes treated with Artemisinin, after 72

hours of incubation

Artemisinin (Chinese-qinghaosu), a sesquiterpene lactone and its derivatives are anti-malaria drug, have demonstrated effectiveness in many experimental research forms of leishmaniasis $(22,23)$. The first study of investigating the leishmanicidal activity of ART (24) and its efficacy against experimental cutaneous leishmaniasis was demonstrated. Although Artemisinin is widely utilized as an anti-malarial agent, it has also shown its anti-promastigote activities and its inhibitory effect on Leishmania was increased(25). Furthermore, Artemisinin proved efficacy on different helminthes in the experimental method including schistosomiasis (25), Trichinellosis (26), clonorchiasis (27) and other helminths (28).

A major number of in-vitro and in-vivo studies have shown that Artemisinin and its derivatives have efficacy in controlling Leishmania species. The drugs showed effectiveness against many protozoan such as Trypanosomae spp., Acanthamoeba spp. and Babesia spp. (29). Previous studies showed that viability of the L. major promastigotes decreased gradually with increasing concentrations of Artemisinin with an $\mathrm{IC}_{50}$ value of $750 \mathrm{nM}$ and 3-30 $\mu \mathrm{M}$ for intracellular amastigotes (25).

The anti-promastigote activity of ART was studied on old and new world leishmaniasis, including $L$. donovani, L. infantum, L. tropica, L. braziliensis, L. mexicana, L. amazonensis respectively. Artemisinin induced a similar group of leishmanicidal efficacy in all species aforementioned, with the $\mathrm{IC}_{50}$ values being $115,120,100,100,120$ and $120 \mu \mathrm{M}$, respectively. The similarity of $\mathrm{IC}_{50}$ values is an evidence of Artemisinin has a broad spectrum of leishmanicidal activity (30). Comparable effects have been observed for the treatment of L. tropica, L. major, L. infantum, L. mexicana, L. braziliensis and L. amazonensis $(31,32)$. 
Artemisinin exhibited high toxicity and apoptotic effect on promastigotes to L. major and lowering development of this disease in BALB/c mice (33). Another study by (38) reinforced all studies former invitro and in-vivo about effects of Artemisinin, they showed that Artemisinin inhibited the growth of L. major promastigotes. Its inhibitory activity toward promastigotes determined the $\mathrm{IC}_{50}$ values of $283 \mu \mathrm{M}$. While in another study on $L$. donovani, the $\mathrm{IC}_{50}$ of Artemisinin was $160 \mu \mathrm{M}$ for promastigotes, which further decreased to $22 \mu \mathrm{M}$ in amastigotes (34). They are effective in inhibiting the parasite metabolism while exhibiting limited adverse effects on the host, indicating a higher safety index of the Artemisinin $(35,36)$.

Another study showed a significant $(\mathrm{P} \leq 0.05)$ decline in the proliferation of amastigotes inside the lesion's macrophages in the Artemisinin treated mice compared with the other macrophages (37). When exposed to microorganisms, (39) demonstrated a blast in oxygen consumption coincident with the production of nitric oxide in large amounts with $\mathrm{H}_{2} \mathrm{O}_{2}$, exhibited more effective leishmanicidal effects (38).

A study by (32) showed that Artemether (derivatives) had an apoptotic effect on L. major promastigotes, and could inhibit the growth of both of promastigotes and amastigotes in-vitro. Artemether is considered a derivative of Artemisinin, had exhibited an anti-leishmanial effect due to the methyl ether derivative of Artemisinin and including high efficacy and low toxicity that could become patented for the therapy of leishmaniasis (39).

The $\mathrm{IC}_{50}$ value of Artemisinin as an anti-malarial drug is in the nanomolar range $(4.2-16.2 \mathrm{nM})$ whereas, for antileishmanial activity, micromolar concentrations are required, However, the safety index of the drug as an anti-leishmanial is high and, as oral, parenteral and rectal dosages can achieve micromolar concentrations (40), Artemisinin could well be considered a potential anti-leishmanial drug. ART compounds have also been shown to inhibit in vitro growth of Trypanosoma cruzi and Trypanosoma brucei subsp. rhodesiense at micromolar concentrations, providing evidence of its expanding therapeutic potential (41).

ART and its derivatives share a common structural feature called endoperoxide linkage (45). Miltefosine is the last new drug which is active in the oral administration. However, miltefosine-resistant parasites have been obtained in laboratory indicating that the miltefosine-resistance is at risk in the field (42). For this reason, the search for new antileishmanial drugs should continue to investigate the antileishmanial activity of compounds having lethal properties on other parasites. Thus, Artemisinin derivatives, dihydroartemisinin(DHA), artesunate, artemether, and Arteether, are currently used for the treatment of malaria in artemisinin combination therapies(43).

\section{Conclusion}

This work is first experiment to investigate the Artemisinin antileishmanial activity on Iraqi strain of $L$. tropica and could be suggested as a promising Therapy for Cutaneous Leishmaniasis, in vivo studies are required to complete this concept.

\section{Acknowledgments}

I am very grateful to Assistant Prof. Dr. Mohammed Mahmood Farhan provide laboratory support to complete the requirements of this research. This study was supported by laboratory of Parasitology in biology department for college of science /University of Baghdad and The Biotechnology Research Center / AL-Nahrain University.

\section{References}

1. Sattarahmady N, Movahedpour A, Heli H, Hatam G. Gold nanoparticles-based biosensing of Leishmania major kDNA genome: visual and spectrophotometric detections. Sensors and Actuators B: Chemical.(2016);235.

2. Wulsten I, Costa-Silva T, Mesquita J, Lima M, Galuppo M, Taniwaki N, et al.. Investigation of the anti-Leishmania Leishmania infantum activity of some natural sesquiterpene lactones. Molecules.(2017);22 (5).

3. Hijjawi N, Kanani KA, Rasheed M, Atoum M, Abdel-Dayem M, Irhimeh MR. Molecular diagnosis and identification of Leishmania species in Jordan from saved dry samples.BioMed research international. (2016).

4. Chappuis F, Sundar S, Hailu A, Ghalib H, Rijal S, Peeling RW, et al.. Visceral leishmaniasis: what are the needs for diagnosis, treatment and control? Nature reviews microbiology. (2007);5 (11supp).

5. Ebrahimisadr P, Ghaffarifar F, Hassan ZM. In-vitro evaluation of antileishmanial activity and toxicity of artemether with focus on its apoptotic effect. Iranian j. of pharmaceutical research: IJPR. (2013);12 (4). 
6. Den Boer M, Davidson RN. Treatment options for visceral leishmaniasis. Expert review of antiinfective therapy.(2006);4 (2).

7. Singh N, Kumar M, Singh RK. Leishmaniasis: current status of available drugs and new potential drug targets. Asian Pacific j. of tropical medicine. (2012);5 (6).

8. Rodrigues IA, Mazotto AM,Cardoso V,Alves RL, Amaral ACF, Silva JRdA, et al.. Natural products: insights into leishmaniasis inflammatory response. Mediators of inflammation. (2015).

9. Hendrickx S, Boulet G, Mondelaers A, Dujardin J, Rijal S, Lachaud L, et al.. Experimental selection of paromomycin and miltefosine resistance in intracellular amastigotes of Leishmania donovani and L. infantum. Parasitology research. (2014);113 (5).

10. Faraut-Gambarelli F, Piarroux R, Deniau M, Giusiano B, Marty P, Michel G, et al.. In vitro and in vivo resistance of Leishmania infantum to meglumine antimoniate: a study of 37 strains collected from patients with visceral leishmaniasis. Antimicrobial agents and chemotherapy. (1997); 41 (4).

11. Coelho AC, Gentil LG, da Silveira JF, Cotrim PC. Characterization of Leishmania (Leishmania) amazonensis promastigotes resistant to pentamidine. Experimental parasitology. (2008); 120 (1).

12. Meshnick SR.Artemisinin: mechanisms of action, resistance and toxicity. International $\mathrm{j}$. for parasitology. (2002);32 (13).

13. Heydari FE, Ghaffarifar F, Soflaei S, Dalimi A. Comparison between in vitro effects of aqueous extract of Artemisia seiberi and artemisinin on Leishmania major. Jundishapur J. of Natural Pharmaceutical Products. (2013);8 (2).

14. Wong $\mathrm{YK}, \mathrm{Xu} \mathrm{C}$, Kalesh KA, He Y, Lin Q, Wong WF, et al.. Artemisinin as an anticancer drug: recent advances in target profiling and mechanisms of action. Medicinal research reviews. (2017); 37 (6).

15. Loo CSN, Lam NSK, Yu D, Su X-z, Lu F. Artemisinin and its derivatives in treating protozoan infections beyond malaria. Pharmacological research. (2017); 117.

16. Berens RL, Brun R, Krassner SM. A simple monophasic medium for axenic culture of hemoflagellates. The J. of parasitology. (1976);62 (3).

17. Kamil MM, Ali HZ. Using PCR for detection of cutaneous leishmaniasis in Baghdad. Iraqi J. of Science. (2016);57 (2B).

18. Efferth T. Cancer combination therapies with artemisinin-type drugs. Biochemical pharmacology. (2017);139, 56-70.

19. Santarem N, Cunha J, Silvestre R, Silva C, Moreira D, Ouellette M, et al.. The impact of distinct culture media in Leishmania infantum biology and infectivity. Parasitology. (2014);141 (2).

20. Heydari FE, Ghaffarifar F, Soflaei S, Dalimi A. Comparison between in vitro effects of aqueous extract of Artemisia seiberi and artemisinin on Leishmania major. Jundishapur J. of Natural Pharmaceutical Products. (2013);8 (2).

21. Quinn GP, Keough MJ. Experimental design and data analysis for biologists, $P$ Cambridge University Press. (2002).

22. Bahuguna A, Khan I, Bajpai VK, Kang SC. MTT assay to evaluate the cytotoxic potential of a drug. Bangladesh J. of Pharmacology. (2017);12 (2).

23. Tayyab Ansari M, Saeed Saify Z, Sultana N, Ahmad I, Saeed-Ul-Hassan S, Tariq I, et al..Malaria and artemisinin derivatives: an updated review. Mini reviews in medicinal chemistry. (2013);13 (13).

24. Want MY, Islamuddin M, Chouhan G, Ozbak HA, Hemeg HA, Dasgupta AK, et al..Therapeutic efficacy of artemisinin-loaded nanoparticles in experimental visceral leishmaniasis.Colloids and Surfaces B:Biointerfaces.(2015);130.

25. Yang D, Liew F. Effects of qinghaosu (artemisinin) and its derivatives on experimental cutaneous leishmaniasis. Parasitology. (1993);106 (1).

26. Danso-Appiah A, Garner $P$, Olliaro $P$, Utzinger J. Treatment of urinary schistosomiasis: methodological issues and research needs identified through a Cochrane systematic review. Parasitology. (2009);136 (13).

27. Rayia DMA, Saad AE, Ashour DS, Oreiby RM. Implication of artemisinin nematocidal activity on experimental trichinellosis: In vitro and in vivo studies. Parasitology international. (2017);66 (2).

28. Keiser J, Shu-Hua X, Jian $X$, Zhen-San C, Odermatt $P$, Tesana S, et al.. Effect of artesunate and artemether against Clonorchis sinensis and Opisthorchis viverrini in rodent models. International $\mathrm{j}$. of antimicrobial agents. (2006); 28 (4).

29. Islamuddin M, Chouhan G, Want MY, Ozbak HA, Hemeg HA, Afrin F. Immunotherapeutic potential of eugenol emulsion in experimental visceral leishmaniasis. PLOS Neglected tropical diseases. (2016); 10 (10).

30. Loo CSN, Lam NSK, Yu D, Su X-z, Lu F. Artemisinin and its derivatives in treating protozoan infections beyond malaria. Pharmacological research. (2017); 117. 
31. Sen R, Ganguly S, Saha P. Chatterjee M.Efficacy of artemisinin in experimental visceral leishmaniasis. International J. of Antimicrobial Agents. (2010);36 (1).

32. Ebrahimisadr P, Ghaffarifar F, Hassan ZM. In-vitro evaluation of antileishmanial activity and toxicity of artemether with focus on its apoptotic effect. Iranian j. of pharmaceutical research: IJPR. (2013); 12 (4).

33. Rodrigues IA, Mazotto AM, Cardoso V, Alves RL, Amaral ACF, Silva JRdA, et al.Natural products: insights into leishmaniasis inflammatory response. Mediators of inflammation. (2015).

34. Ghaffarifar F, Heydari FE, Dalimi A, Hassan ZM, Delavari M, Mikaeiloo H. Evaluation of apoptotic and antileishmanial activities of Artemisinin on promastigotes and BALB/C mice infected with Leishmania major. Iranian j. of parasitology. (2015);10 (2).

35. Ganguly S, Bandyopadhyay S, Bera A, Chatterjee M. Antipromastigote activity of an ethanolic extract of leaves of Artemisia indica. Indian j. of pharmacology. (2006); 38 (1).

36. Sen R, Bandyopadhyay S, Dutta A, Mandal G, Ganguly S, Saha P, et al. Artemisinin triggers induction of cell-cycle arrest and apoptosis in Leishmania donovani promastigotes. J. of medical microbiology. (2007);56(9).

37. Want MY, Islamuddin M, Chouhan G, Ozbak HA, Hemeg HA, Dasgupta AK, et al.Therapeutic efficacy of artemisinin-loaded nanoparticles in experimental visceral leishmaniasis.Colloids and Surfaces B: Biointerfaces.(2015);130.

38. Nemati S, Nahrevanian H, Haniloo A, Farahmand M. Investigation on nitric oxide and C-reactive protein involvement in antileishmanial effects of artemisinin and glucantim on cutaneous leishmaniasis. Adv Studies Biol. (2013); 5.

39. Erel O, Kocyigit A, Bulut V, Gurel MS.Reactive nitrogen and oxygen intermediates in patients with cutaneous leishmaniasis. Memórias do Instituto Oswaldo Cruz. (1999);94 (2).

40. Räth K, Taxis K, Walz G, Gleiter CH, Li S-M, Heide L. Pharmacokinetic study of artemisinin after oral intake of a traditional preparation of Artemisia annua L. (annual wormwood). The American $\mathrm{j}$. of tropical medicine and hygiene. (2004);70 (2).

41. Mishina YV, Krishna S, Haynes RK, Meade JC. Artemisinins inhibit Trypanosoma cruzi and Trypanosoma brucei rhodesiense in vitro growth. Antimicrobial agents and chemotherapy. (2007);51 (5).

42. Chaturvedi D, Goswami A, Saikia PP, Barua NC. Rao PG.Artemisinin and its derivatives: a novel class of anti-malarial and anti-cancer agents. Chemical Society Reviews. (2010); 39 (2).

43. Seifert K, Matu S, Perez-Victoria FJ, Castanys S, Gamarro F, Croft SL.Characterisation of Leishmania donovani promastigotes resistant to hexadecylphosphocholine (miltefosine). International j. of antimicrobial agents. (2003);22 (4).

44. Denis MB, Davis TM, Hewitt S, Incardona S, Nimol K, Fandeur T, et al. Efficacy and safety of dihydroartemisinin-piperaquine (Artekin) in Cambodian children and adults with uncomplicated falciparum malaria. Clinical Infectious Diseases. (2002);35 (12). 